\title{
A combinatorial approach to Macdonald q, t-symmetry via the Carlitz bijection
}

\author{
Maria Monks Gillespie ${ }^{1} \llbracket$ \\ ${ }^{1}$ University of California, Berkeley
}

\begin{abstract}
We investigate the combinatorics of the symmetry relation $\widetilde{H}_{\mu}(\mathbf{x} ; q, t)=\widetilde{H}_{\mu^{*}}(\mathbf{x} ; t, q)$ on the transformed Macdonald polynomials, from the point of view of the combinatorial formula of Haglund, Haiman, and Loehr in terms of the inv and maj statistics on Young diagram fillings. By generalizing the Carlitz bijection on permutations, we provide a purely combinatorial proof of the relation in the case of Hall-Littlewood polynomials $(q=0)$ for the coefficients of the square-free monomials in the variables $\mathbf{x}$. Our work in this case relates the Macdonald inv and maj statistics to the monomial basis of the modules $R_{\mu}$ studied by Garsia and Procesi. We also provide a new proof for the full Macdonald relation in the case when $\mu$ is a hook shape.

Résumé. Nous investiguons la combinatoire de la relation $\widetilde{H}_{\mu}(\mathbf{x} ; q, t)=\widetilde{H}_{\mu^{*}}(\mathbf{x} ; t, q)$ des polynômes (transformés) de Macdonald, du point de vue de la formule de Haglund, Haiman et Loehr, exprimant ces polynômes en termes d'inversions et d'indices majeurs de remplissages de diagrammes de Young. Nous généralisons la bijection de Carlitz sur les permutations, ce qui nous permet de déduire cette relation pour les polynômes de Hall-Littlewood (le cas $q=0$ ), de façon purement combinatoire, pour les monômes sans carrés dans les variables $\mathbf{x}$. Notre approche lie les statistiques de Macdonald inv et maj à la base monomiale des modules $R_{\mu}$ de Garsia-Procesi. Nous fournissons aussi une nouvelle démonstration de la relation (complète) de Macdonald lorsque $\mu$ est une équerre.
\end{abstract}

Keywords. Macdonald polynomials, Hall-Littlewood polynomials, symmetric functions, Young tableaux, cocharge, Mahonian statistics

\section{Introduction}

Let $\Lambda_{q, t}(x)$ denote the ring of symmetric polynomials in the countably many indeterminates $x_{1}, x_{2}, \ldots$, with coefficients in the field $\mathbb{Q}(q, t)$ of rational functions in two variables. The (transformed) Macdonald polynomials $\widetilde{H}_{\mu}(x ; q, t) \in \Lambda_{q, t}(x)$, indexed by the set of all partitions $\mu$, form an orthogonal basis of $\Lambda_{q, t}(x)$, and have specializations $\widetilde{H}_{\mu}(x ; 0,1)=h_{\mu}$ and $\widetilde{H}_{\mu}(x ; 1,1)=e_{1}^{|\mu|}$, where $h_{\lambda}$ and $e_{\lambda}$ are the homogeneous and elementary symmetric functions, respectively. The polynomials $\widetilde{H}_{\mu}$ are a transformation of the functions $P_{\lambda}$ originally defined by Macdonald in [13], and have been the subject of much recent attention in combinatorics and algebraic geometry. [6] [8] [9]

\footnotetext{
${ }^{\dagger}$ Email: monks @math . berkeley. edu. Supported by NSF GRFP and Hertz Foundation. 


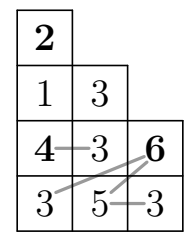

Fig. 1: A filling of a Young diagram. Descents are shown in bold, and attacking pairs are connected by a segment.

The symmetric functions $\widetilde{H}_{\mu}$ may be defined as the unique collection of polynomials that satisfy certain triangularity conditions. However, an explicit combinatorial formula for the transformed Macdonald polynomials $\widetilde{H}_{\mu}$ was discovered in [8] in 2004. The formula is

$$
\widetilde{H}_{\mu}(x ; q, t)=\sum_{\sigma} q^{\operatorname{inv}(\sigma)} t^{\operatorname{maj}(\sigma)} x^{\sigma},
$$

where the sum ranges over all fillings $\sigma$ of the diagram of $\mu$ with positive integers, and $x^{\sigma}$ is the monomial $x_{1}^{m_{1}} x_{2}^{m_{2}} \ldots$ where $m_{i}$ is the number of times the letter $i$ occurs in $\sigma$. The statistics inv and maj are generalizations of the Mahonian statistics inv and maj for permutations. Their precise definitions can be stated as follows.

Definition 1 Given a word $w=w_{1} \cdots w_{n}$ where the letters $w_{i}$ are taken from some partially ordered alphabet $A$, a descent of $w$ is an index $i$ for which $w_{i}>w_{i+1}$. The major index of $w$, denoted maj $(w)$, is the sum of the descents of $w$.

Definition 2 Given a filling $\sigma$ of a Young diagram of shape $\mu$ drawn in French notation ${ }^{(\mathrm{i})}$ let $^{(1)}, \ldots, w^{\left(\mu_{1}\right)}$ be the words formed by the successive columns of $\sigma$, read from top to bottom. Then

$$
\operatorname{maj}(\sigma)=\sum_{s} \operatorname{maj}\left(w^{(s)}\right) .
$$

Example 1 The major index of the filling in Figure 1 is 5, since the first column has major index 4, the second has major index 0 , and the third column, 1.

For the statistic inv, we use the notion of the arm of an entry, defined as the number of squares strictly to the right of the entry. A descent is an entry which is strictly greater than the entry just below it.

Definition 3 An attacking pair in a filling $\sigma$ of a Young diagram is a pair of entries $u$ and $v$ with $u>v$ satisfying one of the following conditions:

1. $u$ and $v$ are in the same row, with $u$ to the left of $v$, or

2. $u$ is in the row above $v$ and strictly to its right.

Definition 4 The quantity $\operatorname{inv}(\sigma)$ is defined as $\operatorname{inv}(\sigma)=\#$ (attacking pairs) $-\sum$ (arms of descents). For a word $w$, the inversion number $\operatorname{inv}(w)$ is equal to inv of the corresponding one-row filling.

\footnotetext{
(i) The Young diagram in Figure 1 has partition shape $\mu=(3,3,2,1)$. French notation indicates that the rows in the diagram have lengths equal to the parts of the partition, listed from bottom to top.
} 
Example 2 In Figure 1 there are 4 attacking pairs, and the arms of the descents have lengths 0, 2, and 0 . Thus $\operatorname{inv}(\sigma)=4-2=2$.

The well-known $q, t$-symmetry relation for the Macdonald polynomials $\widetilde{H}_{\mu}(x ; q, t)$ states that

$$
\widetilde{H}_{\mu}(x ; q, t)=\widetilde{H}_{\mu^{*}}(x ; t, q) .
$$

This is a result of the triangularity conditions that define $\widetilde{H}_{\mu}$, and is also clear from Haiman's geometric interpretation [10]. When combined with the combinatorial formula, we obtain a remarkable identity:

$$
\sum_{\sigma: \mu \rightarrow \mathbb{Z}_{+}} q^{\operatorname{inv}(\sigma)} t^{\operatorname{maj}(\sigma)} x^{\sigma}=\sum_{\rho: \mu^{*} \rightarrow \mathbb{Z}_{+}} q^{\operatorname{maj}(\rho)} t^{\operatorname{inv}(\rho)} x^{\rho} .
$$

Setting $t=1$ and $\mu=(n)$ and taking the coefficient of $x_{1} \cdots x_{n}$ on both sides, this reduces to

$$
\sum_{w \in S_{n}} q^{\operatorname{inv}(w)}=\sum_{w \in S_{n}} q^{\operatorname{maj}(w)}
$$

which demonstrates the well-known equidistribution of the Mahonian statistics inv and maj on permutations. There are several known bijective proofs of this simpler identity (see [1], [3], [14]).

In light of this, it is natural to ask if there is an elementary combinatorial proof of (2), in the sense of Conjecture 1 below. To state the conjecture we first introduce some notation that we will use throughout.

Definition 5 The content of a filling $\sigma$, denoted $|\sigma|$, is the sequence $\alpha=\left(\alpha_{1}, \cdots, \alpha_{k}\right)$ where $\alpha_{i}$ is the number of $i$ 's used in the filling. We also define the symbols:

- $\mathcal{F}$ - set of all fillings of Young diagrams with positive integers

- $\mathcal{F}_{\mu}^{\alpha}$ - set of fillings of shape $\mu$ and content $\alpha$

- $\left.\mathcal{F}_{\mu}^{\alpha}\right|_{\mathrm{inv}=a},\left.\mathcal{F}_{\mu}^{\alpha}\right|_{\text {maj=b }}$ - set of fillings $\sigma \in \mathcal{F}_{\mu}^{\alpha}$ for which $\operatorname{inv}(\sigma)=$ a or $\operatorname{maj}(\sigma)=b$ respectively.

Definition 6 We define a weighted set to be a set $S$ equipped with a list of statistics stat $_{i}: S \rightarrow \mathbb{Z}$, and a morphism of weighted sets to be a map that preserves their statistics. We write $\left(S ; \mathrm{stat}_{1}, \mathrm{stat}_{2}, \ldots\right)$ to denote the weighted set if the statistics are not understood.

Conjecture 1 There is an explicit isomorphism of weighted sets

$$
\varphi:(\mathcal{F} ; \text { inv, maj }) \rightarrow(\mathcal{F} ; \text { maj, inv })
$$

which interchanges inv and maj and sends a partition shape to its conjugate.

In [5], the author provides explicit bijections $\varphi$ for several infinite families of values of $a, b, \alpha$, and $\mu$. Our bijections naturally extend Carlitz's bijection on permutations, which is defined in section 2 In this abridged version, we give bijections in two of the cases explored in [5], and refer to [5] for all proofs.

In Section 3, we give an explicit bijection $\varphi$ in the case that $\mu$ is a hook shape. In Section 4 we investigate the Hall-Littlewood specialization $a=0$, which corresponds to setting $q=0$ in the Macdonald polynomials. We give a bijection in this case for fillings having content $\alpha=(1,1, \ldots, 1)$, and connect our results to the work of Garsia and Procesi [4], and of Killpatrick [11] on the cocharge statistic. 


\section{The Carlitz bijection}

Our approach to the symmetry problem is motivated by Carlitz's bijection $\left(S_{n} ;\right.$ inv $) \rightarrow\left(S_{n} ;\right.$ maj $)$, an alternative to the better-known Foata bijection. A full proof of this bijection can be found in Carlitz's original paper [1], or in a somewhat cleaner form in [14]. We briefly recall the definition here.

Definition 7 A Carlitz code of length $n$ is a word $w=w_{1} \cdots w_{n}$ consisting of nonnegative integers such that $w_{n-i} \leq i$ for all $i$. Let $C_{n}$ denote the set of all Carlitz codes of length $n$, equipped with the combinatorial statistic $\Sigma$ taking a word to the sum of its entries.

Notice that there are $n$ ! Carlitz codes of length $n$. The Carlitz bijection is the composite

$$
\left(S_{n} ; \text { inv }\right) \stackrel{\text { invcode }}{\longrightarrow}\left(C_{n} ; \Sigma\right) \stackrel{\text { majcode }^{-1}}{\longrightarrow}\left(S_{n} ; \text { maj }\right)
$$

of two isomorphisms of weighted sets, defined as follows.

Definition 8 The inversion code of a permutation $\pi$ is the sequence invcode $(\pi)=c_{1}, \ldots, c_{n}$ where $c_{i}$ is the number of attacking pairs, or inversions, of the form $(j, i)$ with $i<j$ and $\pi^{-1}(j)<\pi^{-1}(i)$.

Definition 9 The major index code of a permutation $\pi$, denoted majcode $(\pi)$, is the sequence $c_{1}, \ldots, c_{n}$ defined as follows. Given $\pi \in S_{n}$ written in list notation, successively remove the entries $n, n-1, n-2, \ldots$ and let $c_{i}$ be the amount the major index decreases at the ith step.

Example 3 We have invcode $(4132)=1210$. Indeed, the 1 is the smaller entry of one inversion $(4,1)$, the 2 is the smaller entry of the two inversions $(3,2)$ and $(4,2)$, the 3 is the smaller entry of the inversion $(4,3)$, and the 4 is not the smaller entry of any inversion.

Let $\pi=3241$. We have maj $(\pi)=1+3=4$. Removing the 4 leaves the permutation 321 , which has major index 3 , so $c_{1}=4-3=1$. Removing the 3 results in 21 , which has major index 1 , so $c_{2}=3-1=2$. Continuing in this fashion we find majcode $(\pi)=1210$.

Therefore majcode ${ }^{-1} \circ$ invcode $(4132)=$ majcode $^{-1}(1210)=3241$.

\subsection{Carlitz bijection on words}

We now generalize the Carlitz bijection to words, i.e. to one-column shapes $\mu$ with any content $\alpha$.

Definition 10 Let $A=\left(a_{1}^{\alpha_{1}}, a_{2}^{\alpha_{2}}, \ldots, a_{k}^{\alpha_{k}}\right)$ be any finite multiset of size $n$, with an ordering " $<$ " such that $a_{1}<a_{2}<\cdots<a_{k}$. We say that a word $c$ of length $n$ is $A$-weakly increasing if every subword of the form

$$
c_{\alpha_{1}+\cdots+\alpha_{i}}, c_{\alpha_{1}+\cdots \alpha_{i}+1}, c_{\alpha_{1}+\cdots \alpha_{i}+2}, \ldots, c_{\alpha_{1}+\cdots+\alpha_{i}+\alpha_{i+1}-1}
$$

is weakly increasing.

For instance, if $A=\{1,1,2,3,3,3,4,4\}$, ordered by magnitude, then the word 23711213 is $A$-weakly increasing, since the subwords $23,7,112$, and 13 , corresponding to each letter of $A$, are weakly increasing.

Definition 11 Let $C_{\left(1^{n}\right), A}$ denote the subset of $C_{n}$ consisting of all Carlitz codes of length $n$ which are $A$-weakly increasing. This subset inherits the $\Sigma$ statistic from $C_{n}$.

We also will make use of Macdonald symmetry in the variables $x_{i}$ by defining a bijection on alphabets. 
Definition 12 The reverse of the content $\alpha=\left(\alpha_{1}, \ldots, \alpha_{M}\right)$ is the tuple

$$
r(\alpha)=\left(\alpha_{M}, \alpha_{M-1}, \ldots, \alpha_{1}\right) .
$$

In terms of alphabets, let $A$ be a finite multiset of positive integers with maximum element $M$. The reverse of $A$, denoted $\bar{A}$, is the multiset consisting of the elements $M+1-$ a for all $a \in A$.

We now can define bijections

$$
\text { invcode }:\left(\mathcal{F}_{\left(1^{n}\right)}^{\alpha} ; \text { inv }\right) \rightarrow\left(C_{\left(1^{n}\right), A} ; \Sigma\right) ; \quad \text { majcode }:\left(\mathcal{F}_{(n)}^{r(\alpha)} ; \text { maj }\right) \rightarrow\left(C_{\left(1^{n}\right), A} ; \Sigma\right) .
$$

Definition 13 Let $w$ be a word (corresponding to a filling of a row) consisting of the letters in the ordered alphabet $A=a_{1} \leq \cdots \leq a_{n}$, with ties among the letters broken in the order they appear in $w$. We define $\operatorname{invcode}(w)=c_{1} \cdots c_{n}$ where $c_{i}$ is the number of inversions having $a_{i}$ as the smaller entry.

For example, the inversion code of the filling

$$
\begin{array}{|l|l|l|l|l|l|}
\hline 3 & 2 & 4 & 1 & 3 & 2 \\
\hline
\end{array}
$$

is 313010 , since the 1 is the smaller entry of 3 inversions, the first 2 is the smaller entry of 1 inversion, the second 2 is the smaller entry of 3 inversions, and so on. It will follow from Theorem 2 that:

Proposition 1 The map invcode is an isomorphism of weighted sets invcode : $\mathcal{F}_{\left(1^{n}\right)}^{\alpha} \rightarrow C_{\left(1^{n}\right), A}$.

To define the map majcode, we first require a standardization rule for fillings of columns.

Definition 14 Let $\sigma$ be any filling of a column of height $n$ with positive integers. We define the standardization labeling on repeated entries as follows. Let $i$ be a letter that occurs $k$ times in $\sigma$.

1. Remove all entries larger than $i$ to form a smaller column $\sigma^{\prime}$.

2. Assign a label of $k$ to the bottommost $i$ that is either at the very bottom of $\sigma^{\prime}$ or for which the entries $a$ and $b$ directly north and south of it satisfy $a>b$. Repeat this process, labeling the next $i$ by $k-1$ and so on, until there are no i's that satisfy this condition.

3. Remove and label the remaining $i$ 's from top to bottom, decreasing the label by 1 each time.

We define Standardize $(\sigma)$ to be the unique column filling using labels $1,2, \ldots, n$ that respects the ordering of the entries of $\sigma$ and breaks ties according to the standardization labeling.

Proposition 2 For any column filling $\sigma$ with alphabet $A$, let $\rho=\operatorname{Standardize}(\sigma)$. Then $\rho$ and $\sigma$ have the same major index, and majcode $(\rho)$ is A-weakly increasing.

We now can define the map majcode on words, that is, for one-column fillings.

Definition 15 Let $\sigma$ be any filling of a column shape $\mu=\left(1^{r}\right)$. We define

$$
\operatorname{majcode}(\sigma)=\operatorname{majcode}(\operatorname{Standardize}(\sigma)),
$$

where majcode of a standard filling is defined to be the majcode of its reading word.

Proposition 3 The map majcode is a weighted set isomorphism $\mathcal{F}_{\left(1^{n}\right)}^{r(\alpha)} \rightarrow C_{\left(1^{n}\right), A}$ for any alphabet $A$ with content $\alpha$, and any one-column partition shape $\left(1^{n}\right)$.

See the full paper [5] for proofs of Propositions 1, 2, and 3. 


\section{Hook Shapes}

We now demonstrate a new bijective proof of Conjecture 1 in the case that $\mu$ is a hook shape, that is, $\mu=(m, 1,1,1, \ldots, 1)$ for some $m$. The symmetry of inv and maj was demonstrated for fillings of hook shapes having distinct entries in [2], and makes use of the Foata bijection. In this section, we instead give a generalization the Carlitz bijection to this setting, which will hold for arbitrary fillings by the results in Section 2.1.

Definition 16 Let $\sigma$ be a filling of a hook shape $\mu$. We define the hook codes of $\sigma$ to be the pair of codes consisting of the invcode of its bottom row and the majcode of its leftmost column, along with the data of which entries occur in the row and which occur in the column.

To extend the standardization orderings on the row and column of $\mu$ as defined in Section 2.1, if the corner square in $\mu$ is one of the repeated letters $a$ of the filling, then we consider it the largest $a$ in its column and the smallest $a$ in its row. Thus we can define a standardization ordering on fillings of hook shapes: we order the letters smallest to largest, with the following tie-breaking rules.

- If two copies of the letter $a$ appear in the (leftmost) column, the tie is broken as in Definition 14

- If they appear in the (bottom) row, then the leftmost $a$ comes first.

- If one appears in the column and the other in the row, the $a$ in the column comes first.

This enables us to represent hook codes as a table, as shown in the following example.

Example 4 Consider the filling $\sigma$ of a hook shape shown below. The 2 in the corner is considered to be greater than the 2 above it and less than the 2 to its right. To represent the hook code of $\sigma$, we write the entries of the filling in the standardization ordering, and write the invcode and (the reverse of) majcode of the bottom row and left column respectively underneath the corresponding letters.

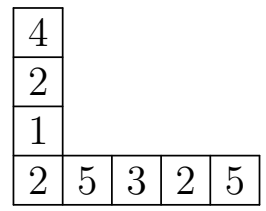

\begin{tabular}{lllllllll} 
& 1 & 2 & 2 & 2 & 3 & 4 & 5 & 5 \\
\hline invcode & & & 0 & 2 & 1 & & 0 & 0 \\
majcode & 0 & 1 & 0 & & & 2 & &
\end{tabular}

Notice that the majcode is written backwards, because the entries are in increasing order.

We can now define our bijection.

Definition 17 For any hook shape $\mu$ and content $\alpha$, let $\phi: \mathcal{F}_{\mu}^{\alpha} \rightarrow \mathcal{F}_{\mu^{*}}^{r(\alpha)}$ be the map defined by interchanging the pair of hook codes of a filling and writing them backwards, and also reversing its alphabet.

Example 5 Starting with the tableau in Example 4 if we reverse the alphabet, interchange invcode and majcode, and write the codes in backwards order, then we obtain the filling and pair of codes below. It follows that the filling in Example 4 maps to the filling below under $\phi$. 


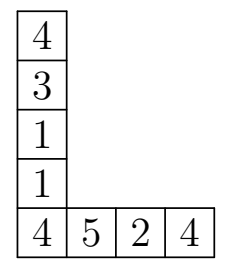

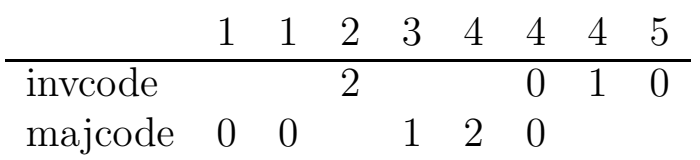

In [5] we show that $\phi$ is indeed a weight-preserving bijection on hook shapes:

Theorem 1 We have that

$$
\operatorname{maj}(\phi(\sigma))=\operatorname{inv}(\sigma) \quad \text { and } \quad \operatorname{inv}(\phi(\sigma))=\operatorname{maj}(\sigma)
$$

for any filling $\sigma$ of a given hook shape $\mu$. Moreover, $\phi$ is a bijection from $\mathcal{F}_{\mu}^{\alpha}$ to $\mathcal{F}_{\mu^{*}}^{r(\alpha)}$ for any content $\alpha$.

\section{Hall-Littlewood Specialization at $q=0$}

We now turn to the specialization in which one of the statistics is zero. In particular, setting $q=0$, the symmetry relation becomes $\widetilde{H}_{\mu}(x ; 0, t)=\widetilde{H}_{\mu^{*}}(x ; t, 0)$, which is a symmetry relation on the transformed Hall-Littlewood polynomials $\widetilde{H}_{\mu}(x ; t):=\widetilde{H}_{\mu}(x ; 0, t)$. In this case the identity becomes

$$
\sum_{\substack{\sigma: \mu \rightarrow \mathbb{Z}_{+} \\ \operatorname{inv}(\sigma)=0}} t^{\operatorname{maj}(\sigma)} x^{\sigma}=\sum_{\substack{\rho: \mu^{*} \rightarrow \mathbb{Z}_{+} \\ \operatorname{maj}(\rho)=0}} t^{\operatorname{inv}(\rho)} x^{\rho} .
$$

Combinatorially, we wish to find natural morphisms

$$
\varphi:\left.\left.\mathcal{F}_{\mu}^{\alpha}\right|_{\text {inv }=0} \rightarrow \mathcal{F}_{\mu^{*}}^{r(\alpha)}\right|_{\text {maj=0 }}
$$

of weighted sets, where $\left.\mathcal{F}_{\mu}^{\alpha}\right|_{\text {inv=0 }}$ is equipped with the maj statistic, and $\left.\mathcal{F}_{\mu^{*}}^{\alpha}\right|_{\text {maj=0 }}$ is equipped with the inv statistic. For the bijection $r(\alpha)$, we will use the reverse map of Definition 12

In the case that $\operatorname{inv}(\sigma)=0$, the statistic maj is essentially the same as the cocharge statistic defined by Lascoux and Schützenberger in [12]. This connection lies in the cocharge word construction (Figure 2). One of the key steps towards understanding this is the following:

Proposition 4 Let $\mu=\left(\mu_{1}, \ldots, \mu_{k}\right)$ be a partition. Given a tuple of multisets $\left(A_{1}, \ldots, A_{k}\right)$ of positive integers where $\left|A_{i}\right|=\mu_{i}$ for all $i$, there is a unique filling $\sigma$ of $\mu$ with $\operatorname{inv}(\sigma)=0$ whose ith row contains precisely the numbers in $A_{i}$ for all $i$.

Proof: Since $\operatorname{inv}(\sigma)=0$, the bottom row has the elements of $A_{1}$ in increasing order from left to right. We now induct on the rows. Suppose row $i$ is filled in with entries $b_{1}, \ldots, b_{r}$ left to right. The leftmost entry $a_{1}$ of row $i+1$ must be the smallest element of $A_{i+1}$ that comes after $b_{1}$ in "cyclic order" (i.e. either larger than $b_{1}$, or $\min A_{i+1}$ if $\left.b_{1}>\max A_{i+1}\right)$. Then the next entry $a_{2}$ must be the smallest element of $A_{i+1} \backslash\left\{a_{1}\right\}$, that comes after $b_{2}$ in cyclic order, and so on. This uniquely determines row $i+1$.

Definition 18 The cocharge word of a filling $\sigma: \mu \rightarrow \mathbb{Z}_{+}$is the word $\mathrm{cw}(\sigma)=i_{1} i_{2} \cdots i_{n}$ consisting of the row heights of the cells $u_{k}=\left(i_{k}, j_{k}\right)$, where $u_{1}, u_{2}, \ldots, u_{n}$ is the ordering of the cells of $\mu$ such that $\sigma\left(u_{1}\right) \geq \sigma\left(u_{2}\right) \geq \cdots \geq \sigma\left(u_{n}\right)$, with ties broken in reverse reading order. 
In [8], it was shown that for any filling $\left.\sigma \in \mathcal{F}\right|_{\text {inv }=0}$ we have

$$
\operatorname{maj}(\sigma)=\operatorname{cocharge}(\operatorname{cw}(\sigma))
$$

where cocharge is the statistic defined in [12]. It also turns out that any filling with no inv can be uniquely reconstructed from its cocharge word and its alphabet. This easily shown using the notion of a relative inversion defined in [5].

In what follows, we will only be considering the case $\alpha=\left(1^{n}\right)$, i.e. fillings having distinct entries $1, \ldots, n$. For the general case see [5].

\subsection{Generalized Carlitz Codes}

We now generalize Carlitz codes to fillings having inv or maj equal to 0 , and content $\alpha=\left(1^{n}\right)$. Our generalization is motivated by the monomial basis of the Garsia-Procesi modules in [4], which are closely connected to the cocharge (maj) statistic.

Definition 19 A word having letters in $\{0,1,2, \ldots\}$ is Yamanouchi if every suffix contains at least as many i's as $i+1$ 's for all $i \geq 0$. A word $w=w_{1} \cdots w_{n}$ is $\mu$-sub-Yamanouchi, or $\mu$-Carlitz, if there exists a Yamanouchi word $v=v_{1} \cdots v_{n}$ of content $\mu$ such that $w_{i}<v_{i}$ for all $i$.

Example 6 The sub-Yamanouchi words for $\mu=(1,1,1, \ldots, 1)$ are precisely the classical Carlitz codes.

Definition 20 We write $C_{\mu}$ for the set of all $\mu$-sub-Yamanouchi codes, equipped with the sum statistic $\Sigma$.

We now introduce the concept of the monomial of a code. The next three definitions are compatible with the notation in [4].

Definition 21 Fix variables $x_{1}, x_{2}, \ldots$ For any finite code $c$ of length $n$, define its monomial to be

$$
x^{c}=x_{n}^{c_{1}} x_{n-1}^{c_{2}} \cdots x_{1}^{c_{n}} .
$$

Also let $\mathcal{C}(\mu)$ be the set of all monomials $x^{c}$ of $\mu$-sub-Yamanouchi words $c$.

In [4], the authors define a similar set of monomials $\mathcal{B}(\mu)$, which are the generators of the $S_{n}$-modules $R_{\mu}$ whose Frobenius characteristics are precisely the Hall-Littlewood polynomials. In [5], we show that $\mathcal{C}(\mu)=\mathcal{B}(\mu)$, by showing that the sets $\mathcal{C}(\mu)$ satisfy the recursion in [4]. To state this recursion we require two more definitions, which follow the notation in [4].

Definition 22 Given a partition $\mu$, define $\mu^{(i)}$ to be the partition formed by removing the unique corner square whose row has the same length as the ith row $\mu_{i}$.

\begin{tabular}{|l|l|l|l|l|}
\cline { 1 - 3 } $\mathbf{7}$ & 1 & $\mathbf{4}$ & \multicolumn{2}{|}{} \\
\cline { 1 - 4 } $\mathbf{5}$ & $\mathbf{6}$ & 1 & 2 & \multicolumn{1}{|c}{} \\
\hline 2 & 3 & 5 & 5 & 8 \\
\hline
\end{tabular}

Fig. 2: A filling $\sigma$ with cocharge word 132112311223. It is the unique filling with its given sets of row entries that has no inversions, by Proposition 4 This allows us to recover $\sigma$ from its cocharge word and alphabet. 
Definition 23 Given a set of monomials $\mathcal{C}$ and a monomial $m$, we write $m \cdot \mathcal{C}$ to denote the set of all monomials of the form $m \cdot x$ where $x \in \mathcal{C}$.

Definition 24 The sets $\mathcal{B}(\mu)$ are defined by $\mathcal{B}((1))=\{1\}$ and the recursion

$$
\mathcal{B}(\mu)=\bigsqcup_{i-1}^{\mu_{1}^{*}} x_{n}^{i-1} \cdot \mathcal{B}\left(\mu^{(i)}\right) .
$$

Proposition 5 The sets $\mathcal{C}(\mu)$ satisfy the same recursion as $\mathcal{B}(\mu)$, and $\mathcal{C}_{\{1\}}((1))=\left\{x_{1}\right\}=\mathcal{B}((1))$.

This shows that $\mathcal{C}(\mu)=\mathcal{B}(\mu)$, as claimed.

\subsection{Inversion Codes}

We can now generalize the inversion code of a permutation to arbitrary fillings $\rho$ with maj $(\rho)=0$.

Definition 25 Let $\rho$ be a filling of $\mu^{*}$ having maj $(\rho)=0$. Order its entries by size with ties broken in reading order to form a totally ordered alphabet $\left\{a_{1}, \ldots, a_{n}\right\}$. Then its inversion code is the sequence invcode $(\rho)=c_{1} \cdots c_{n}$ whose ith entry $c_{i}$ is the number of attacking pairs having $a_{i}$ as its smaller entry.

Example 7 There are three attacking pairs, as shown, in the filling below.

$$
\begin{array}{|l|l|}
\hline 1 & 1 \\
\hline 1 & 3 \\
\hline 2 & 3-1 \\
\hline
\end{array}
$$

Its inversion code is 0002100. Indeed, to compute the invcode, we order the entries by size and reading order and record the number of attacking pairs having each entry as the smaller of the pair:

\begin{tabular}{l|lllllll} 
Entries & 1 & 1 & 1 & 1 & 2 & 3 & 3 \\
\hline Code & 0 & 0 & 0 & 2 & 1 & 0 & 0
\end{tabular}

Theorem 2 The inversion code of any filling $\rho \in \mathcal{F}_{\mu^{*}}^{\alpha}$ is $\alpha$-weakly increasing and $\mu$-sub-Yamanouchi. Moreover, the map

$$
\text { invcode : }\left.\mathcal{F}_{\mu^{*}}^{\alpha}\right|_{\text {maj=0 }} \rightarrow C_{\mu, A}
$$

is an isomorphism of weighted sets, where $C_{\mu, A}$ is the set of $\mu$-sub-Yamanouchi words that are A-weakly increasing. In particular invcode restricts to a bijection $\left.\mathcal{F}_{\mu^{*}}^{\left(1^{n}\right)}\right|_{\text {maj=0 }} \rightarrow C_{\mu}$.

See [5] for the full proof of this result.

\subsection{Major Index Codes}

To complete the proof of the Hall-Littlewood case with $\alpha=\left(1^{n}\right)$, it now suffices to find a weighted set isomorphism

$$
\text { majcode }:\left(\left.\mathcal{F}_{\mu}^{\left(1^{n}\right)}\right|_{\text {inv=0 }} ; \text { maj }\right) \rightarrow\left(C_{\mu}, \Sigma\right) \text {. }
$$

By Proposition 5, the $\mu$-sub-Yamanouchi monomials satisfy the recursion $\mathcal{C}(\mu)=\bigsqcup_{i=1}^{\mu_{1}^{*}} x_{n}^{i-1} \cdot \mathcal{C}\left(\mu^{(i)}\right)$. It now suffices to show that $\left.\mathcal{F}_{\mu}^{\left(1^{n}\right)}\right|_{\text {inv=0 }}$ satisfies a similar recursion, with its maj statistic recorded by the 
exponents of the monomials. In particular, we first need an analog of the notion of "removing the largest entry" as in the Carlitz bijection. We wish to find an explicit weighted set isomorphism

$$
\psi:\left(\left.\mathcal{F}_{\mu}^{\left(1^{n}\right)}\right|_{\text {inv }=0} ; \text { maj }\right) \rightarrow \bigsqcup_{d}\left(\left.\mathcal{F}_{\mu^{(d+1)}}^{\left(1^{n-1}\right)}\right|_{\text {inv }=0} ; \text { maj }+d\right),
$$

so that $\psi$ sends an inversion-free filling $T$ of $\mu$ to an inversion-free filling $\psi(T)$ of $\mu^{(d+1)}$ for some $d$, such that $\operatorname{maj}(\psi(T))=\operatorname{maj}(T)-d$. Then if we define majcode $(T)=d_{1} d_{2} \ldots d_{n}$ where $d_{k}=$ $\operatorname{maj}\left(\psi^{k}(T)\right)-\operatorname{maj}\left(\psi^{k-1}(T)\right)$, we are done.

Such a map can be extracted from the work of Killpatrick [11], which gives a combinatorial proof of a recursion for a generating function involving charge. The charge statistic, written ch, is related to the cocharge by the equation $\operatorname{ch}(\mu)=n(\mu)-\operatorname{cocharge}(\mu)$ where $n(\mu)=\sum_{i}(i-1) \cdot \mu_{i}$. Killpatrick defines $W_{\mu}$ to be the set of words of content $\mu$, and lets $r_{i, \mu}=\left|\left\{j>i: \mu_{j}=\mu_{i}\right\}\right|$. The recursion is stated as:

$$
\sum_{w \in W_{\mu}} q^{\operatorname{ch}(w)}=\sum_{i} q^{r_{i, \mu}} \sum_{w \in W_{\mu}(i)} q^{\operatorname{ch}(w)}
$$

If we substitute $q \rightarrow 1 / q$ and multiply both sides by $q^{n(\mu)}$, this becomes

$$
\sum_{w \in W_{\mu}} q^{\operatorname{cocharge}(w)}=\sum_{i} q^{i-1} \sum_{w \in W_{\mu}^{(i)}} q^{\operatorname{cocharge}(w)},
$$

which is essentially the same as the recursion for $\mu$-sub-Yamanouchi codes. Killpatrick's work, when translated into the language of fillings via the cocharge word construction, gives a map $\psi$ as in Equation (4). We now give a translated description of the map $\psi$.

Definition 26 The crank of a filling $\sigma$ having $\operatorname{inv}(\sigma)=0$ and alphabet $\{1, \ldots, n\}$ is the filling formed by (a) decreasing each entry $i \geq 2$ by 1 and replacing the entry 1 with $n$, and (b) rearranging the entries within each row in the unique way such that inv $=0$, as in Proposition 4 .

Definition 27 A crank orbit $i$ s the set of all fillings obtained by repeatedly applying the crank to a filling.

Note that the crank orbits have sizes $|\mu| / d$ where $d$ is some common divisor of the parts of $\mu$. Furthermore, the crank orbits partition the fillings of $\mu$ into disjoint subsets. (See Figure 3 .)

Let $T$ be a filling with $\operatorname{inv}(T)=0$. We now give a five-step algorithm for computing $\psi(T)$, and throughout we use the example

$$
T=\begin{array}{|l|l|}
\hline 3 & 4 \\
\hline 1 & 2 \\
\hline
\end{array}
$$

Step 1: List the crank orbits and mark the "special" fillings. List the entries of each crank orbit for $\mu$ in order by starting with a filling and repeatedly applying the crank. We define a special filling of such an orbit to be a filling for which the largest entry $n=|\mu|$ occurs in the bottom row of the tableau.

Step 2: Assign difference values plus one to the special fillings. For each special filling $\sigma^{\star}$, define

$$
\left.\operatorname{diff}\left(\sigma^{\star}\right)=\operatorname{maj}\left(\operatorname{crank}^{-1}\left(\sigma^{\star}\right)\right)\right)-\operatorname{maj}\left(\sigma^{\star}\right)
$$

Assign to each special filling the number

$$
\ell\left(\sigma^{\star}\right)=\operatorname{diff}\left(\sigma^{\star}\right)+1 .
$$




\begin{tabular}{|c|c|c|c|c|c|c|}
\hline & \begin{tabular}{l|l}
1 & 2 \\
\end{tabular} & \begin{tabular}{l|l}
4 & 1 \\
\end{tabular} & \begin{tabular}{l|l}
3 & 4 \\
\end{tabular} & \begin{tabular}{l|l}
2 & 3 \\
\end{tabular} & \begin{tabular}{l|l|}
3 & 1 \\
\end{tabular} & \begin{tabular}{l|l}
2 & 4 \\
\end{tabular} \\
\hline & \begin{tabular}{l|l}
3 & 4
\end{tabular} & \begin{tabular}{l|l}
2 & 3
\end{tabular} & \begin{tabular}{|l|l}
1 & 2 \\
\end{tabular} & \begin{tabular}{l|l}
1 & 4 \\
\end{tabular} & \begin{tabular}{l|l|}
2 & 4 \\
\end{tabular} & \begin{tabular}{l|l}
1 & 3 \\
\end{tabular} \\
\hline special? & * & & & $\star$ & $\star$ & \\
\hline maj & 0 & 1 & 2 & 1 & 1 & 2 \\
\hline$\ell\left(\sigma^{\star}\right)$ & 2 & & & 2 & 2 & \\
\hline label & $z_{1}$ & $z_{1}$ & $z_{2}$ & $z_{2}$ & $z_{1}$ & $z_{1}$ \\
\hline
\end{tabular}

Fig. 3: The crank orbits for $\mu=(2,2)$, along with their special entries, maj and $\ell$ values, and labels.

The work in [11] shows that the values $\ell\left(\sigma^{\star}\right)$, ranging over all special fillings in a given orbit of size $|\mu| / d$, will be the sizes of the column lengths of the partition $\mu / d$ formed by dividing each of the rows of $\mu$ by $d$.

Step 3: Assign labelings. Starting with any special filling $\sigma^{\star}$ in a given orbit, label it $z_{1}$ and proceed to label each entry in the orbit according to the following algorithm. Continue labeling entries by $z_{1}$ in order until we have either labeled $\ell\left(\sigma_{1}^{\star}\right)$ of them, or until we encounter another special filling $\sigma_{2}^{\star}$. In either case, change our label to $z_{2}$ and start labeling entries with $z_{2}$ starting from $\sigma_{2}^{\star}$ in the same manner. If we finish labeling $\ell\left(\sigma_{2}^{\star}\right)$ fillings with $a_{2}$ and have not finished labeling with $z_{1}$ 's, return to $z_{1}$ until it is finished or we reach the next star, which we label $z_{3}$, and so on. (See Figure 3.) It turns out that we will end up with the same partition of the orbit given by the labels $z_{i}$ no matter which special filling that we start at. [11]

Step 4: Sort the labeled entries into columns. For the crank orbit of $T$, sort all of the entries labeled $z_{1}$, all those labeled $z_{2}$, and so on each into their own column with the special entry at the bottom and the rest above it in the order they appear in the orbit. This forms a set of columns which, if arranged in decreasing order of height, forms the partition shape $\mu / d$.

For example, for the first orbit in Figure 3 , we have two columns:

$$
\begin{aligned}
& \begin{array}{|l|l|}
\hline 4 & 1 \\
\hline 2 & 3 \\
\hline
\end{array} \begin{array}{|l|l|}
\hline 3 & 4 \\
\hline 1 & 2 \\
\hline
\end{array} \\
& \begin{array}{|l|l|}
\hline 1 & 2 \\
\hline 3 & 4 \\
\hline
\end{array} \quad \begin{array}{|l|l|}
\hline 2 & 3 \\
\hline 1 & 4 \\
\hline
\end{array}
\end{aligned}
$$

Step 5: Bumping from the bottom. Find the location of $T$ in one of the columns produced in Step 4. Let $i$ be the number of fillings below $T$ in its column. Let $T^{\star}$ be the special filling in the bottom row of the column of $T$. Let cell $c=\left(\right.$ row, col) be the corner of $T^{\star}$ that is removed to form shape $\mu^{(i)}$, and let $T_{\rightarrow}^{\star}$ be the filling formed by deleting columns $1, \ldots$, col -1 of $T^{\star}$. So, in the running example, $T$ is in the upper right corner of the diagram from Step 4, and so $c=2, i=1, T^{\star}=$\begin{tabular}{|l|l|}
\hline 2 & 3 \\
\hline 1 & 4
\end{tabular} and $T_{\rightarrow}^{\star}=$\begin{tabular}{|l|}
3 \\
\hline 4
\end{tabular} .

Define the bumping sequence to be the sequence of entries $b_{\text {row }}, b_{\text {row }-1}, \ldots, b_{1}$ where $b_{\text {row }}$ is the entry in square $c$, and for all $j, b_{j}$ is the entry in row $j$ of $T_{\rightarrow}^{\star}$ which is the largest entry less than $b_{j+1}$ (or the very largest entry, if $b_{j+1}$ is less than all entries in row $j$ ). It turns out that we always have $b_{1}=n$.

Finally, remove $b_{1}=n$ and bump down $b_{1}, \ldots, b_{\text {row }}$ one row each. Re-order the rows so that there are no inversions, and the resulting tableau is $\psi(T)$. In the running example we have $\psi(T)=$\begin{tabular}{|l|l|}
2 \\
\hline 1 & 3 \\
\hline
\end{tabular} . Killpatrick's proof shows that maj $(\psi(T))=\operatorname{maj}(T)-i$. It follows that $\psi$ gives rise to a map majcode that completes the proof of symmetry in this case.

Theorem 3 In the case $\alpha=\left(1^{n}\right)$ of fillings with distinct entries, we have that $\varphi=$ majcode ${ }^{-1} \circ$ invcode 
is an isomorphism of weighted sets

$$
\varphi:\left.\left.\mathcal{F}_{\mu}^{\left(1^{n}\right)}\right|_{\mathrm{maj}=0} \rightarrow \mathcal{F}_{\mu^{*}}^{\left(1^{n}\right)}\right|_{\mathrm{inv}=0} .
$$

\section{Acknowledgments}

The author thanks Mark Haiman and Angela Hicks for many insightful conversations. Thanks also to the referee for typo corrections and helpful feedback.

\section{References}

[1] L. Carlitz, A combinatorial property of $q$-Eulerian numbers, The American Mathematical Monthly, Vol. 82, No. 1 (Jan. 1975), pp. 51-54.

[2] G. Davis, A. Maurer, J. Michelman, An Open Problem in the Combinatorics of Macdonald Polynomials, Carleton College, https://apps.carleton.edu/curricular/math/assets/compspaper.pdf.

[3] D. Foata and M. Schützenberger, Major Index and Inversion Number of Permutations, Math. Nachr. 83 (1978), pp. 143-159.

[4] A. M. Garsia, C. Procesi, On certain graded $S_{n}$-modules and the $q$-Kostka polynomials, Advances in Mathematics, Vol. 94, Issue 1 (Jul. 1992), pp. 82-138.

[5] M. Gillespie, A combinatorial approach to the $q, t$-symmetry relation in Macdonald polynomials, submitted to Electronic Journal of Combinatorics, June 2015, arXiv:1503.02109.

[6] J. Haglund, The $q, t$-Catalan Numbers and the Space of Diagonal Harmonics: With an Appendix on the Combinatorics of Macdonald Polynomials, University Lecture Series, Vol. 41 (2008).

[7] J. Haglund, The genesis of the Macdonald polynomial statistics, Séminaire Lotharingien de Combinatoire 54A (2006).

[8] J. Haglund, M. Haiman, N. Loehr, A combinatorial formula for Macdonald polynomials, J. Amer. Math. Soc. 18 (2005), 735-761.

[9] M. Haiman, Combinatorics, Symmetric Functions, and Hilbert Schemes, Current Developments in Mathematics, No. 1 (2002), 39-111.

[10] M. Haiman, Hilbert schemes, polygraphs, and the Macdonald positivity conjecture, J. Amer. Math. Soc., 14 (2001), 941-1006.

[11] K. Killpatrick, A combinatorial proof of a recursion for the $q$-Kostka Polynomials, Journal of Combinatorial Theory Series A, Volume 92 Issue 1 (Oct. 2000), 29-53.

[12] A. Lascoux, M. Schützenberger, Sur une conjecture de H.O. Foulkes, C. R. Acad. Sci. Paris Sér A-B, 286 (7), A323-A324, 1978.

[13] I. Macdonald, Symmetric functions and Hall polynomials, Oxford University Press, 1979.

[14] M. Skandera, An Eulerian partner for inversions, Séminaire Lotharingien de Combinatoire 46 (2001), Article B46d. 